\title{
Viscosity Transient Phenomenon in Drop Impact Testing of Soft Material
}

\author{
Chen Liu, Yukio Fujimoto, Yoshikazu Tanaka, Eiji Shintaku, Toshiki Nakanishi \\ Department of Transportation and Environmental Engineering, Graduate School of Engineering, \\ Hiroshima University, Higashi-Hiroshima, Japan \\ Email: fujimoto@naoe.hiroshima-u.ac.jp
}

Received 1 April 2016; accepted 6 May 2016; published 9 May 2016

Copyright (C) 2016 by authors and Scientific Research Publishing Inc.

This work is licensed under the Creative Commons Attribution International License (CC BY). http://creativecommons.org/licenses/by/4.0/

(c) (i) Open Access

\begin{abstract}
The authors carried out drop impact tests for several soft materials under a flat frontal impact condition in which a drop hammer with a flat bottom surface strikes a plate-like soft material in the normal direction. The experimental results indicated that the impact force waveforms of soft materials consisted of a thorn-shaped waveform and a subsequent mountain-shaped waveform. The thorn-shaped waveform was strongly affected by the strain rate. In the present study, the occurrence mechanism of this distinctive waveform was discussed from the viewpoint of the viscosity transient phenomenon. A standard linear solid (SLS) model in which the viscosity transient phenomenon was considered was applied to the simulation. Some features of the impact force waveform of soft materials could be explained by the SLS model. Furthermore, the thorn-shape waveform could also be observed in the impact force waveforms of human skin and free-falling hollow balls.
\end{abstract}

\section{Keywords}

Strain Rate Dependence, Soft Material, Impact Force, Thorn-Shape Waveform, Viscosity Transient, Standard Linear Solid (SLS) Model, Flat Frontal Impact

\section{Introduction}

The authors develop an impact force sensor that can accurately measure a high-speed impact force waveform and apply the sensor to impact tests of several soft materials. Experiments are carried out using compact drop test equipment under a flat frontal impact condition [1]-[3].

In a flat frontal impact, a free-falling drop hammer with a flat bottom surface strikes a plate-like soft material in the normal direction. Synchronized impact forces are measured using two sensors installed on both the drop hammer side and the floor side. The examined soft materials are a sponge plate, a sponge rubber plate, a gel 
plate, a silicon rubber plate, a nitrile rubber plate, tofu, konjac, an oil clay plate, a low-rebound urethane foam plate, a cork plate, sliced ham, pork ham steak, and pork. With the exception of the cork plate, all of these materials exhibit a thorn-shaped waveform and a subsequent mountain-shaped waveform [1]-[3].

In the impact testing of soft materials, a thorn-shaped waveform (spike-like feature) has appeared in previous experiments [4]-[6]. Song et al. concluded that this feature was a result of the radial inertia effect [4]. However, the authors believe that this unique waveform is a result of the double-strike phenomenon caused by the viscosity transient phenomenon [3]. When a soft material receives an impact force, a large viscous resistance is induced at the beginning of the impact. The viscous resistance then decreases rapidly with time. Furthermore, the authors demonstrate that the feature of the impact force waveform can be qualitatively simulated by a standard linear solid (SLS) model [2]. In the SLS model, the damping coefficient of a dashpot is treated as time dependent.

In the present study, the influences of strain rate and impact area on the thorn-shaped waveform are discussed based on the SLS model and the experimental results.

\section{Impact Force Waveform of Soft Materials under Flat Frontal Impact}

Figure 1(a) shows a typical impact force waveform of soft material under a flat frontal impact [1]-[3]. Figure 1(b) summarizes the feature of thorn-shaped waveforms obtained by the experimental observations. The impact force waveform of soft materials is the mixture of two types of waveforms. The first (thorn-shaped) waveform shows a large viscosity resistance (red isosceles triangle Q), and the second (mountain-shaped) waveform shows a small viscosity resistance (dashed black curve R). This resistance change occurs suddenly at the thorn peak. In other words, a viscosity transient is induced at the thorn peak. The soft material surface is struck twice by the relative movement of the colliding objects.

The thorn-shaped waveform has a steep slope on the rising segment (which is $\theta$ ), which usually approximates a straight line. The $\theta$ and the thorn peak become large as the strain rate increase. The second part of the waveform has a gentler slope (which is $\alpha$ ) and is mountain shaped. The slope $\alpha$ becomes gentler than the initial slope $\theta$ because a viscosity discontinuity is induced by the first strike.

\section{Compact Drop Test Equipment and Condition of Flat Frontal Impact}

Figure 2 shows the free-fall drop test equipment used in the present study. The drop hammer is made of an aluminum circular disk plate, a stainless cylinder weight, and a steel bar (total mass: $1.9 \mathrm{~kg}$ ). The contact surface of the hammer bottom is a disk plate having a diameter of $D=60 \mathrm{~mm}$. The upper sensor is an orbicular pad sensor and is attached near the bottom of the drop hammer. The lower sensor is a $100 \mathrm{~mm} \times 100 \mathrm{~mm}$ pad sensor and is attached to the bottom of the frame. Both sensors are made from piezoelectric film. A high-speed voltage recorder with a sampling rate of $20 \mathrm{kHz}$ to $200 \mathrm{kHz}$ is used to record the sensor output. The output of the upper sensor is modified by $8 \%$ in order to account for the mass of the disk plate. Rigorous tests using a servo-controlled testing machine and a high-rigidity load cell confirmed that both sensors can measure the impact force very accurately when the contact time is in the range of $0.1 \mathrm{~ms}$ to $2 \mathrm{~s}$. The drop height $h$ is the height from the upper surface of the soft material to the bottom surface of the drop hammer.

The soft materials are square shaped and their surface areas are larger than the area of drop hammer bottom.

(Q): Thorn-shaped waveform

(R): Mountain-shaped waveform

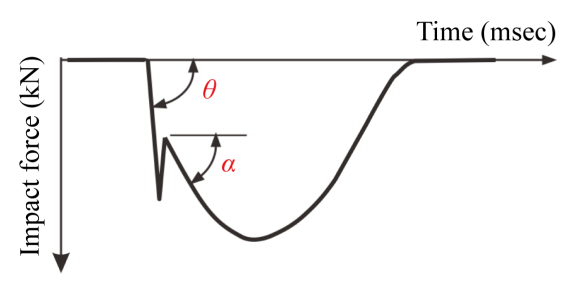

(a)

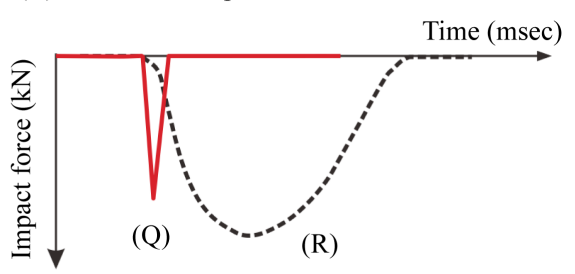

(b)

Figure 1. (a) Typical impact force waveform of soft material under a flat frontal impact. (b) Two types of waveforms contained in the impact force waveform of soft material. 


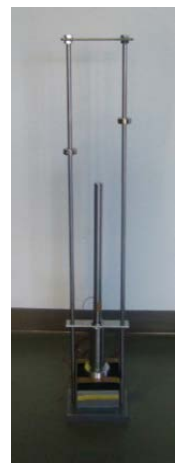

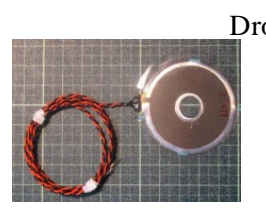

Upper sensor

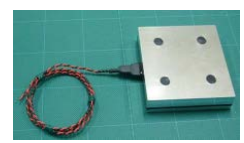

Lower sensor

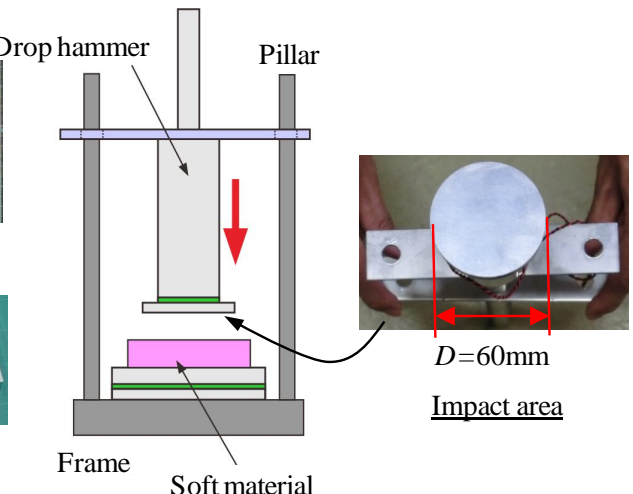

Figure 2. Drop test equipment and the upper and lower sensors used in the device.

\section{Simulation of Impact Force Waveform by the SLS Model}

Figure 3 shows the condition under which the drop weight (mass: $m=1.9 \mathrm{~kg}$ ) collides with a plate-like soft material at speed $V_{0}$. The thickness of the soft material is $T$. The soft material is modeled by the SLS model. The mass (drop weight) is not connected to the spring and dashpot. The SLS model adds an elastic spring $k_{1}$ to the Maxwell model (a viscous damper $c$ and an elastic spring $k_{2}$ ) in parallel. The impact velocity $V_{0}$ is given to the mass as the initial condition. The differential equation of the SLS model is given as Equation (1), where $x_{2}$ is the displacement of the hammer position, and $x_{1}$ is the displacement of the dashpot. The initial strain rate is $\dot{\varepsilon}_{0}=$ $V_{0} / T\left(\mathrm{~s}^{-1}\right)$. The Runge-Kutta method is used for solving the equation of motion. The time increment in the calculation is $5 \times 10^{-6} \mathrm{~s}$. The software was developed by the authors using Visual Basic.

$$
\left\{\begin{array}{l}
m \ddot{x}_{2}+k_{1} x_{2}+k_{2}\left(x_{2}-x_{1}\right)=0 \\
k_{2}\left(x_{2}-x_{1}\right)=c \dot{x}_{1}
\end{array}\right.
$$

The spring coefficients $k_{1}$ and $k_{2}$ are assumed to be constant throughout the collision. As mentioned above, we believe that the viscosity transient occurs during the early stage of the impact force. This influence is taken into consideration through the damping coefficient $c$ of the dashpot, which is treated as a time-dependent value.

\section{Comparison of Experimental and Simulation Results}

\subsection{Influence of Impact Velocity on the Thorn-Shaped Waveform}

Figure 4 compares the impact force waveforms of the upper sensor measured by compact drop test equipment. The soft material is a nitrile rubber (NBR) square plate with one side $100 \mathrm{~mm}$. The thickness is $T=30 \mathrm{~mm}$ (rubber hardness: 70). The drop height is $h=100,200$, or $380 \mathrm{~mm}$. The impact velocity is $V_{0}=1.4 \mathrm{~m} / \mathrm{s}$ when $h=$ $0.1 \mathrm{~m}$ (strain rate: $\left.\dot{\varepsilon}_{0}=V_{0} / T=46 \mathrm{~s}^{-1}\right), V_{0}=1.98 \mathrm{~m} / \mathrm{s}$ when $h=0.2 \mathrm{~m}\left(\dot{\varepsilon}_{0}=66 \mathrm{~s}^{-1}\right)$, and $V_{0}=2.73 \mathrm{~m} / \mathrm{s}$ when $h$ $=0.38 \mathrm{~m}\left(\dot{\varepsilon}_{0}=91 \mathrm{~s}^{-1}\right)$. A thorn shape is clearly observed in the rising segment of the waveform. In the experimental figure, when the drop height $h$ becomes large, the slope $\theta$ of the rising segment becomes steeper and the height of the thorn peak becomes larger. The heights of thorn peak and mountain peak are roughly proportional with the impact velocity $V_{0}$.

Figure 5 shows the impact force waveforms simulated by the SLS model for the three cases. Figure 6 shows the values of the assumed parameters in the SLS model. The spring coefficients are $k_{1}=1000 \mathrm{kN} / \mathrm{m}$ and $k_{2}=$ $3000 \mathrm{kN} / \mathrm{m}$. The damping coefficient $c$ of the dashpot is assumed to be a time-dependent value, which changes during the impact period. When $0<t<0.2 \mathrm{~ms}, c=2000 \mathrm{~kg} / \mathrm{s}$. During the period $0.2<t<0.4 \mathrm{~ms}, c$ decreases linearly from $2000 \mathrm{~kg} / \mathrm{s}$ to $300 \mathrm{~kg} / \mathrm{s}$. During the period $t>0.4 \mathrm{~ms}, c=300 \mathrm{~kg} / \mathrm{s}$. The period $0.2<t<0.4 \mathrm{~ms}$ is the period of the viscosity transient.

The simulation was conducted by varying $k_{1}, k_{2}$, and $c$ in order to search for the condition in which the simulated waveform becomes close to the experimental waveform. In other words, $k_{1}, k_{2}$, and $c$ are decided such that the thorn height, the subsequent mountain height, and the total impact period of the waveform match those of the experiments. 


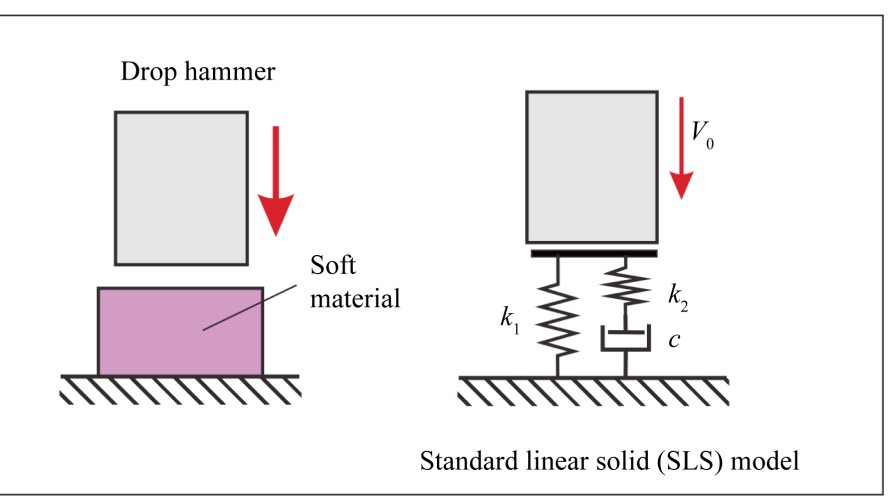

Figure 3. SLS model for the simulation of impact force waveform of soft material.

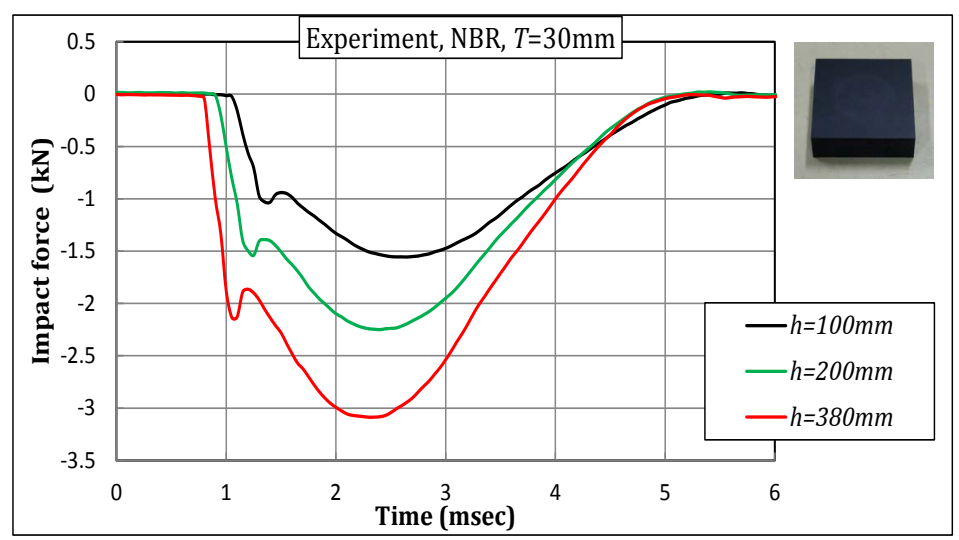

Figure 4. Impact force waveform measured by the weight drop onto NBR plate with $T=30 \mathrm{~mm}$.

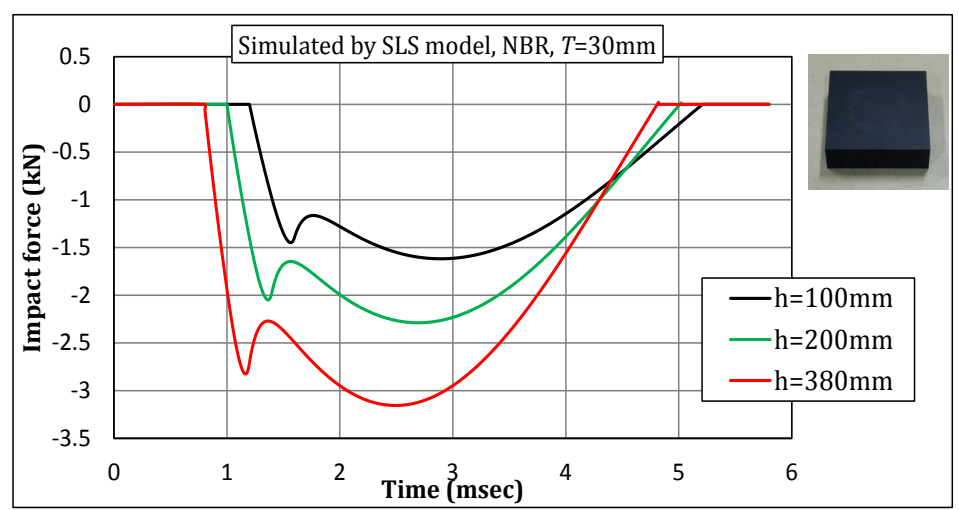

Figure 5. Impact force waveform of NBR plate simulated by the SLS model.

A thorn-shaped waveform is clearly observed in the simulation. Note that, in the simulation, the slope $\theta$ of the thorn becomes steeper and the thorn height becomes larger according to the increase in the impact velocity $V_{0}$ (or strain rate $\dot{\varepsilon}_{0}$ ). The total impact period is approximately the same as that of the experiments. The height of the thorn peak is roughly proportional to the impact velocity $V_{0}\left(=h^{1 / 2}\right)$ in both the experiments and the simulation. In other words, the shape of the thorn is related to the strain rate of the soft material.

In Figure 5, the impulse of the simulated waveform is larger than that of the experimental waveform. One reason for this difference is considered to be that the SLS model cannot exactly represent the coefficient of restitution of the drop hammer after the collision. 


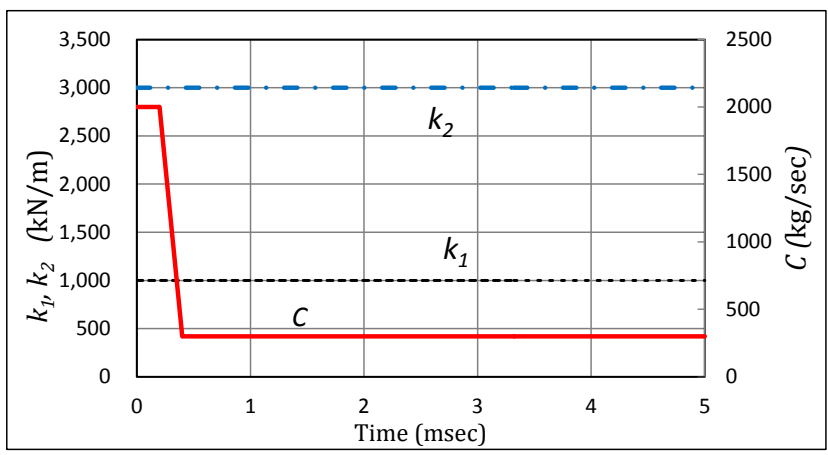

Figure 6. Spring coefficients $k_{1}$ and $k_{2}$ and damping coefficient $c$ assumed for the NBR plate with $T=30 \mathrm{~mm}$.

\subsection{Influence of Impact Area on the Thorn-Shaped Waveform}

Circular aluminum plates having a diameter of $D=20,30,40$, or $50 \mathrm{~mm}$ and a thickness of $d=5$ to $8 \mathrm{~mm}$ was attached to the bottom of the drop hammer, as shown in Figure 7. The hammer was dropped from a height of $h$ $=380 \mathrm{~mm}$ onto a $30 \mathrm{~mm}$-thick NBR plate. The purpose of this experiment was to determine how the impact force waveform is influenced by the impact area. Since the circular plate is thick, the impact force acts only on the circular plate and does not reach the bottom of the drop hammer.

Figure 8 shows the measured impact waveforms. Solid curves correspond to the upper sensor, and dashed curves correspond to the lower sensor. Since the diameter of the aluminum disk is small, the disk is buried deeply into the NBR. Therefore, according to the reduction of the impact area, the contact time of the waveform becomes long and the peak force becomes small. In addition, the thorn peak becomes lower as the impact area becomes smaller. This suggests that the thorn becomes unclear for the case in which the drop hammer has a spherical bottom surface.

Figure 9 shows the impact force waveforms simulated by the SLS model. The assumed $k_{1}, k_{2}$, and $c$ are summarized in Table 1. The values of $k_{1}, k_{2}$, and $c$ in Figure 6 are assumed for the case of $D=60 \mathrm{~mm}$. Then, the values of $k_{1}, k_{2}$, and $c$ are proportionally changed in accordance with the respective impact area.

In Figure 9, the thorn height decreases as the impact area becomes smaller. Moreover, the impact time increases as the impact area becomes smaller. In other words, the simulation results qualitatively represent the characteristics of the experimental results.

\subsection{Case of an Oil Clay Plate under a Flat Frontal Impact}

Figure 10(a) shows the impact force waveform (as measured by the upper sensor) of an oil clay plate with 100 $\mathrm{mm}$ square. Its thickness is about $T=50 \mathrm{~mm}$. The drop heights are $h=100$ and $530 \mathrm{~mm}$. A large thorn is observed. As the drop height increases ( $V_{0}$ becomes higher), the slope $\theta$ becomes steeper and thorn peak becomes higher. The thorns are more obvious than those for other materials. As shown in the enlarged view, the shape of the thorn is similar to an isosceles triangle.

Figure 10(b) shows the waveforms simulated by the SLS model. Here, $k_{1}$ is assumed to be equal to $40 \mathrm{kN} / \mathrm{m}$, and $k_{2}$ is assumed to be equal to $800 \mathrm{kN} / \mathrm{m}$. The damping coefficient $c$ of the dashpot is assumed to be a timedependent value, which changes during the impact period.

Table 2 shows the spring and damping coefficients of the oil clay. During the period of $0<t<0.2 \mathrm{~ms}, c=$ $1500 \mathrm{~kg} / \mathrm{s}$. During the period of $0.2<t<0.5 \mathrm{~ms}, c$ decreases linearly from $1500 \mathrm{~kg} / \mathrm{s}$ to $100 \mathrm{~kg} / \mathrm{s}$. During the period of $t>0.5 \mathrm{~ms}, c=100 \mathrm{~kg} / \mathrm{s}$. The period $0.2<t<0.5 \mathrm{~ms}$ is the period during which the viscosity transient occurs. The spring coefficients are $k_{1}=30 \mathrm{kN} / \mathrm{m}$ and $k_{2}=800 \mathrm{kN} / \mathrm{m}$.

\section{Discussion}

Figure 11 shows the two types of collision condition under a flat frontal impact. Condition 1 is the case that the soft material surface is larger than the area of drop hammer bottom. Condition 2 is the case that the soft material surface is smaller than the area of drop hammer bottom. All the previous our experiments were carried out under 


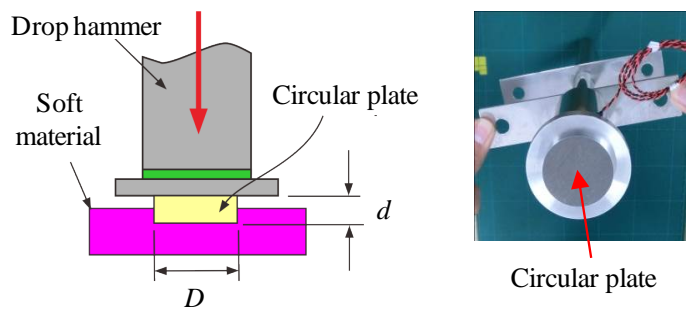

Figure 7. Circular aluminum plate attached to the bottom of the drop weight.

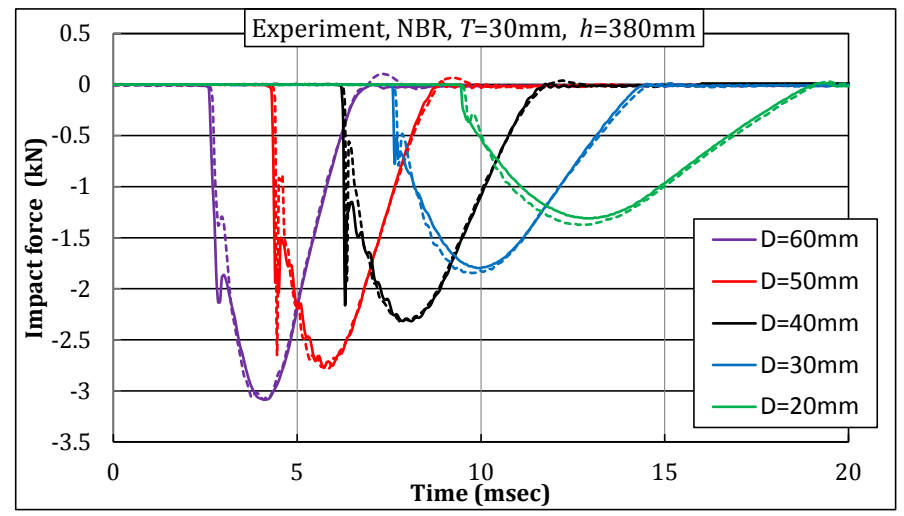

Figure 8. Impact force waveforms measured by the weight drop onto NBR plate with $T=30 \mathrm{~mm}$.

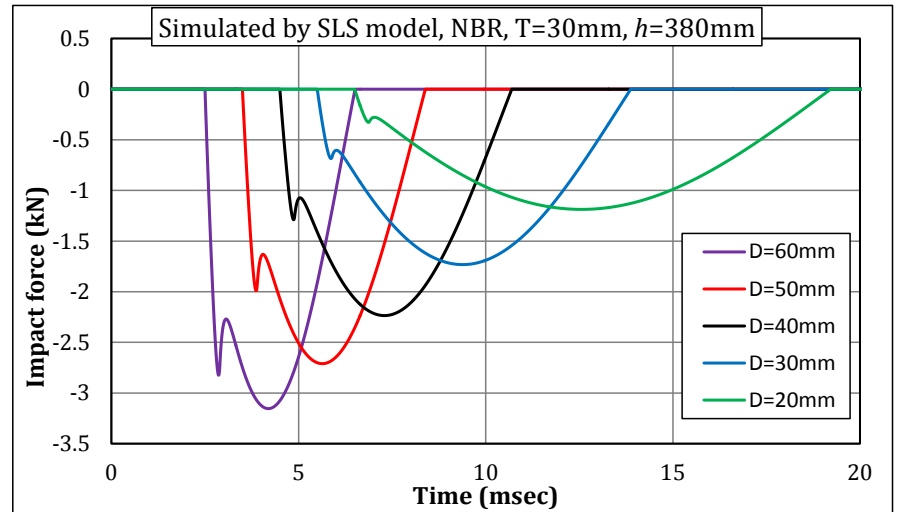

Figure 9. Impact force waveforms of NBR plate simulated by SLS model.

Table 1. Spring coefficients and damping coefficients of NBR assumed in the SLS model.

\begin{tabular}{|c|c|c|c|c|c|c|}
\hline \multirow{2}{*}{$\begin{array}{c}\text { Diameter of AL plate } \\
\qquad D(\mathrm{~mm})\end{array}$} & \multirow{2}{*}{$\begin{array}{l}\text { Impact area } \\
A\left(\mathrm{~mm}^{2}\right)\end{array}$} & \multicolumn{2}{|c|}{ Spring coefficient } & \multicolumn{3}{|c|}{ Damping coefficient $c(\mathrm{~kg} / \mathrm{sec})$} \\
\hline & & $k_{1}(\mathrm{kN} / \mathrm{m})$ & $k_{2}(\mathrm{kN} / \mathrm{m})$ & $0<t<0.2$ msec & $0.2<t<0.4$ msec & $t>0.4 \mathrm{msec}$ \\
\hline 60 & 2827 & 1000 & 3000 & 2000 & Linearly decrease & 300 \\
\hline 50 & 1963 & 694 & 2083 & 1389 & Linearly decrease & 208 \\
\hline 40 & 1257 & 444 & 1333 & 889 & Linearly decrease & 133 \\
\hline 30 & 707 & 250 & 750 & 378 & Linearly decrease & 75 \\
\hline 20 & 315 & 111 & 333 & 222 & Linearly decrease & 33 \\
\hline
\end{tabular}


Table 2. Spring coefficients and damping coefficient of oil clay plate assumed in the SLS model.

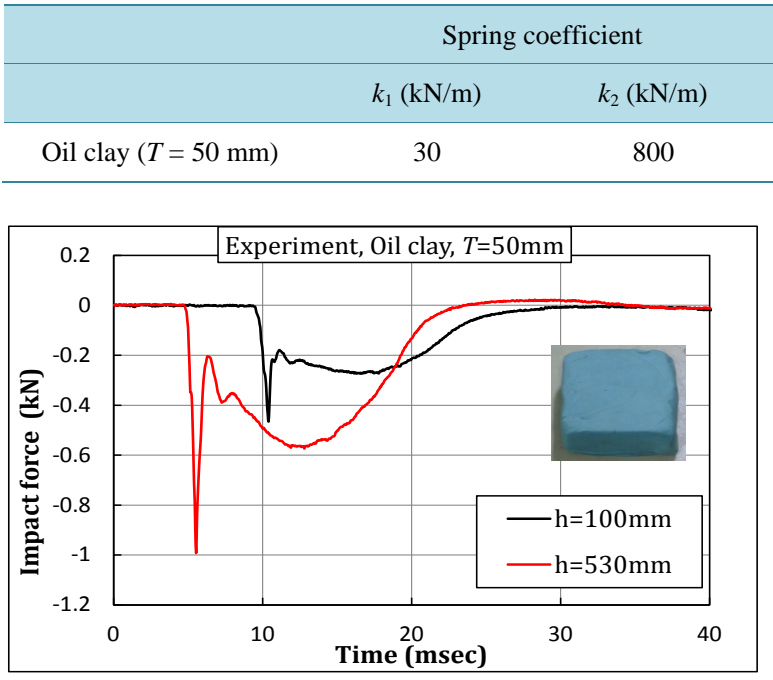

(a)

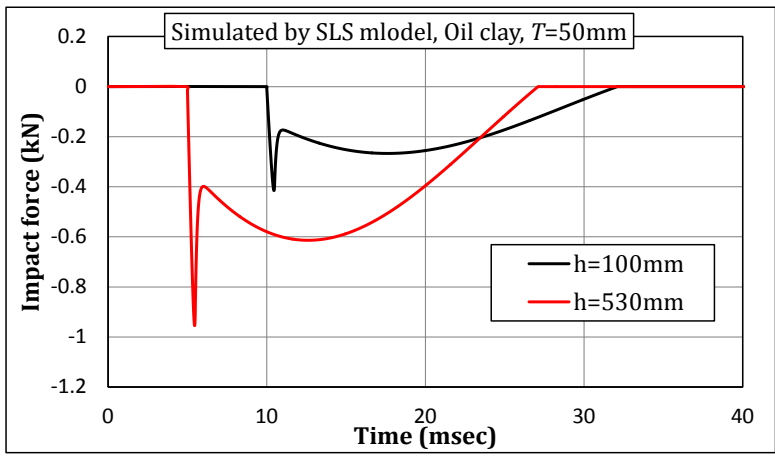

(b)

Figure 10. Impact force waveforms of oil clay plate measured experimentally and simulated by SLS model.

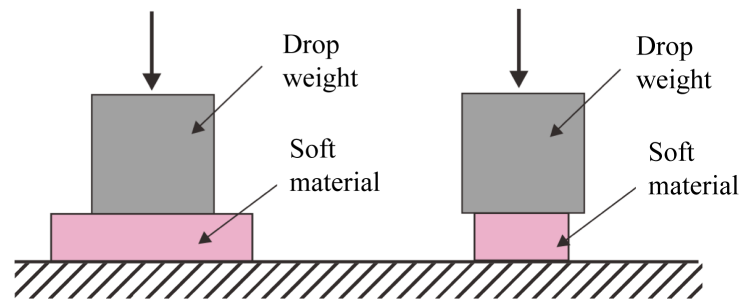

Condition 1

Condition 2

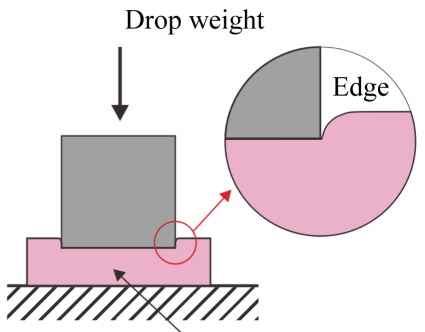

Soft material

Figure 11. Two types of collision conditions under flat frontal impact.

the Condition 1. In the Condition 1, the surrounding soft material near the edge of the drop weight bottom is momentarily deformed by the impact. This edge effect may be the cause of the thorn.

So, we conducted the experiment under the Condition 2. An NBR circle plate with $50 \mathrm{~mm}$ diameter which is smaller than the area of drop hammer bottom is used for the experiment. The thickness of NBR is $T=30 \mathrm{~mm}$ and the drop height is $h=100,200$, or $380 \mathrm{~mm}$.

Figure 12 shows the impact force waveforms of the upper sensor measured by the compact drop test equipment. As is seen in the figure, the thorn-shaped waveform became smaller than that of Figure 4. However, the thorns are still observed in the rising segment of the waveform.

Another experiment of Condition 2 was carried out using an oil cray circle plate with $50 \mathrm{~mm}$ diameter. The thickness of oil cray is $T=50 \mathrm{~mm}$ and the drop height was $h=100$ or $530 \mathrm{~mm}$. Figure 13 shows the impact force waveforms. Although the height of the thorn became smaller, thorn-shaped waveform still occurred.

Why does this small thorn occur? The author thought that it may be a friction effect between the drop weight bottom and the soft material surface. Usually, soft material deforms into a barrel shape by the frictional forces induced in the upper and lower surface of the soft material. So, we conducted an experiment with paint machine oil to the upper and lower surfaces of NBR circle plate. However, the thorn-shaped waveform which did not change so much was appeared for all the cases.

Oil clay contains two components: a liquid (oil) and a solid. Therefore, the viscous resistance of oil may initially appear as a thorn-shaped waveform, followed by a mountain-shaped waveform indicting the resistance of the solid. However, the thorn-shaped waveforms also occur in NBR which does not contain liquid. Therefore, the physical reason for the occurrence of the thorn is unknown at present.

From this fact, the authors are thinking the followings reasons. 


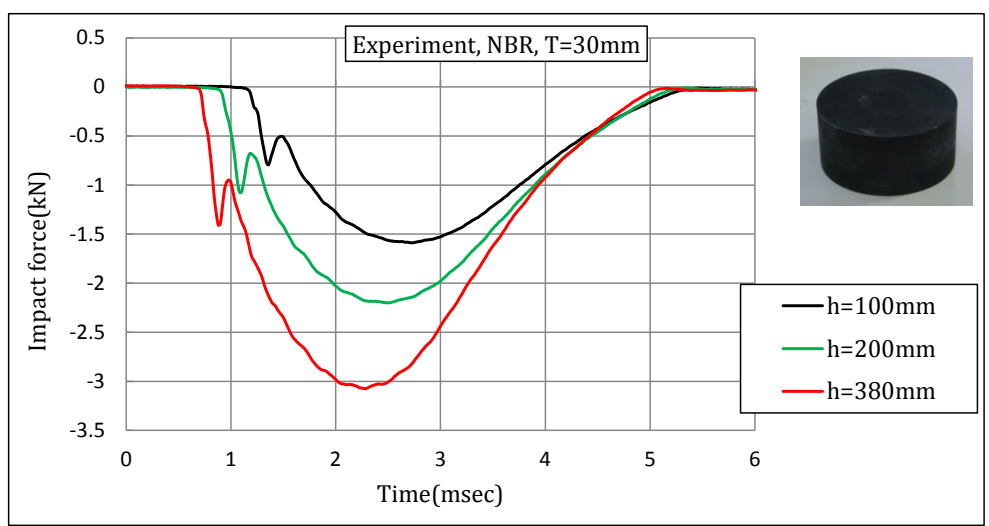

Figure 12. Impact force waveforms measured by the weight drop onto NBR plate with $T=30 \mathrm{~mm}$.

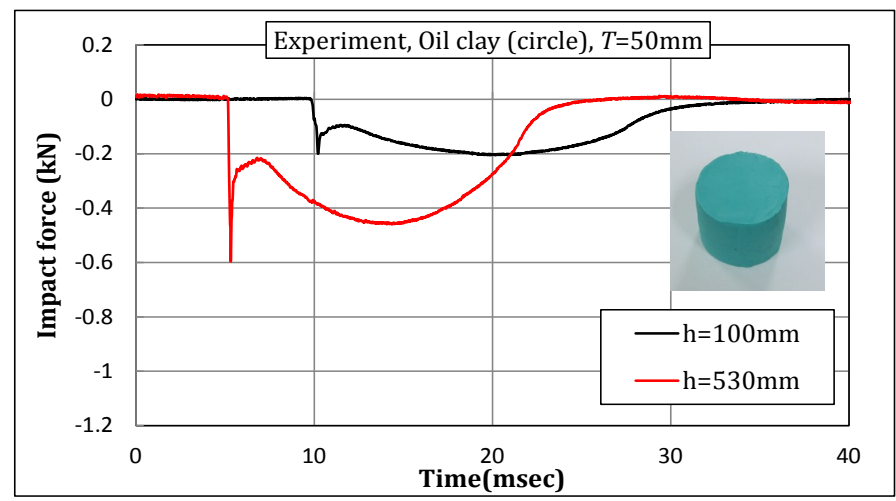

Figure 13. Impact force waveforms measured by the weight drop onto oil clay plate with $T=50 \mathrm{~mm}$.

- Discontinuous viscosity behavior similar with some colloidal suspensions [7] [8] may occur at the thorn peak.

- Breaking of surface tension of soft material may occur at the thorn peak.

- Internal friction may change from static friction to dynamic friction at the thorn peak.

\section{Impact Force of Human Skin under a Flat Frontal Impact}

The drop hammer was removed from the compact drop test equipment, and the hammer was held by hand, as shown in Figure 14. Then, the relatively smooth areas of forearm skin, calf skin, and thigh skin were struck by the hand-held hammer. Considering the above mentioned results, a thorn-shaped waveform should be observed on flat skin areas without bone directly underneath. Impact force waveforms with steep slopes $\theta$ in the rising segment and small thorns were observed in most cases.

\section{Impact Force of Free-Falling Hollow Balls}

Figure 15 shows the impact force waveform of a superball (solid ball) with an excellent rebound property, as measured through free-fall drop tests from heights of $1.0 \mathrm{~m}$ and $2.0 \mathrm{~m}$. No thorn appears in the waveform. Similarly, the impact forces of a hardball (used for baseball), a softball, a golf ball, and a bowling ball (solid balls) did not generate thorn-shaped waveforms due to the spherical surfaces of the balls.

Figure 16 shows the waveforms of a handball, a rubber ball, and a tennis ball (hollow balls) obtained through free-fall drop tests. Even though they have spherical surfaces, the hollow balls generate a steep slope $\theta$ and a small thorn. This is thought to be the reason that the contact area of a hollow ball is flattened at the moment of impact, and a condition similar to a flat frontal impact is achieved. 

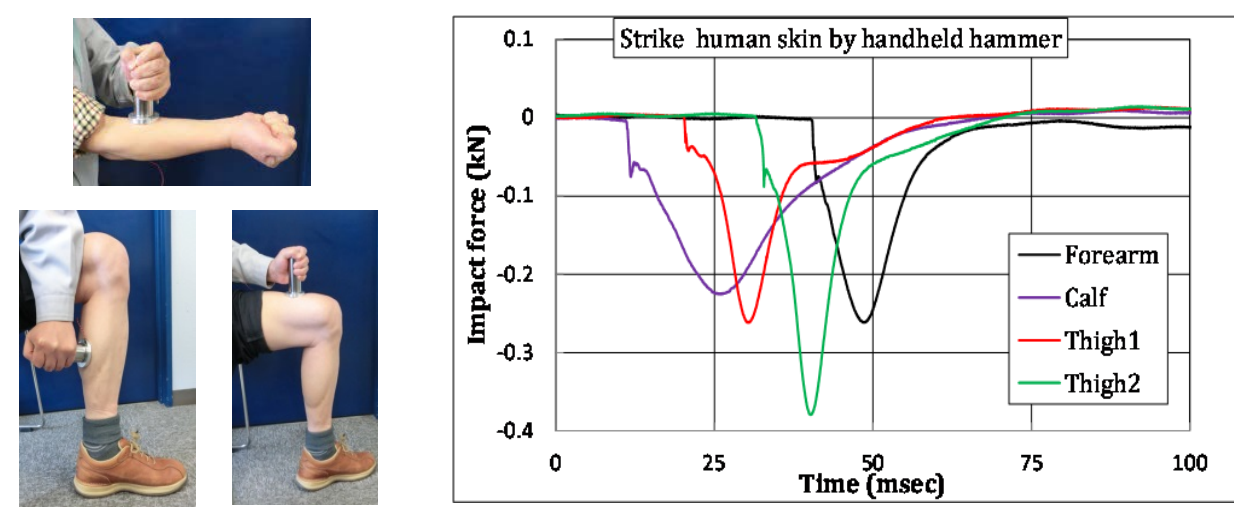

Figure 14. Human skin was struck by a hand-held hammer. The measured impact force waveforms are shown on the right.
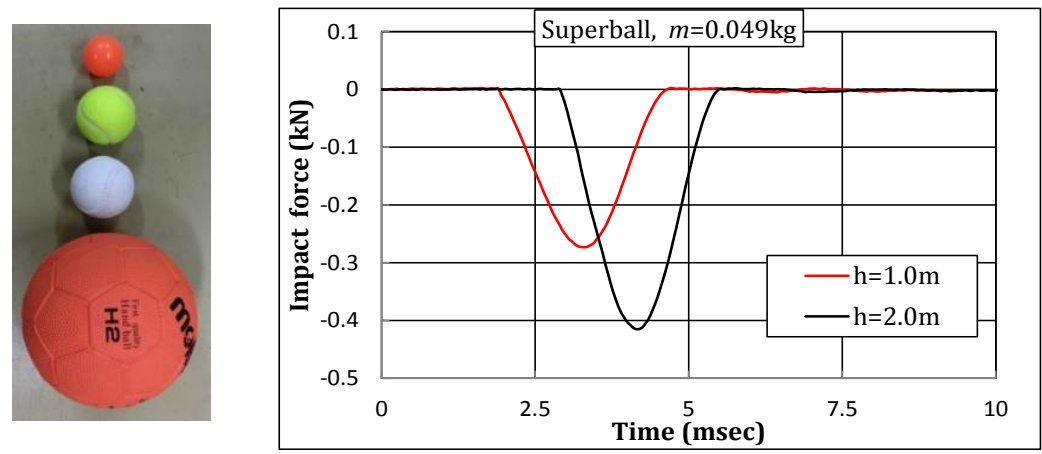

Figure 15. Impact force induced by free-falling superball (solid ball).

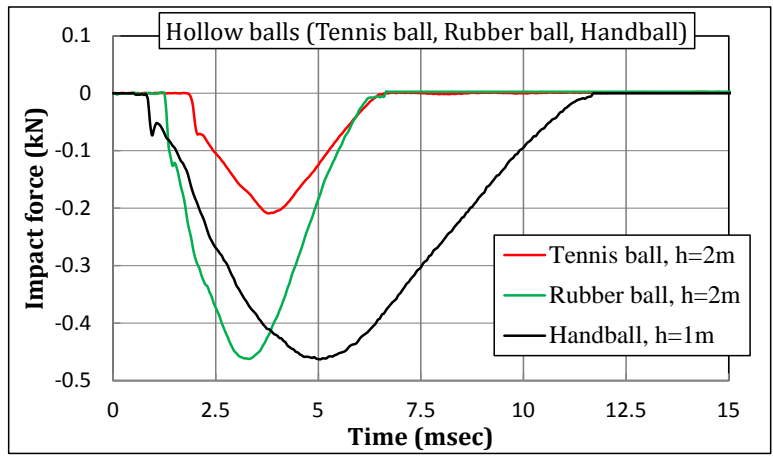

Figure 16. Impact force induced by free-falling hollow balls.

\section{Conclusions}

The impact force waveforms of soft materials were examined based on a drop impact experiment and a dynamics model. Experiments were carried out using compact drop test equipment under the condition of a flat frontal impact. The standard linear solid model (SLS model), in which viscosity transient was considered, was used for the simulation of the impact force waveform. The results obtained here were as follows:

1. The impact force waveforms of soft material consist of a thorn-shaped waveform and a subsequent mountain-shaped waveform. The thorn-shaped waveform is influenced by the strain rate. The rising slope $\theta$ of the thorn becomes steeper as the strain rate increases. The height of the thorn peak becomes higher as the strain rate increases.

2. The SLS model can qualitatively simulate the characteristics of thorn-shaped waveforms.

3. Under the condition of a flat frontal impact, the thorn appears clearly according with the increase in the 
impact area. If either the surface of the drop hammer or the surface of the soft material is curved, the thorn-shaped waveform becomes unclear. These conditions can be reproduced in the simulation using the SLS model.

4. From the experimental observations, the authors think that viscosity transient is induced at the thorn peak. At the moment of impact, a large viscous resistance is induced near the surface of soft material, and the viscous resistance is rapidly reduced after generation of the thorn. However, the physical reason of the viscosity transient phenomenon is unclear.

\section{Acknowledgements}

We thank the Editor and the referee for their comments. The authors also thank to the Venture Business Laboratory of Hiroshima University for the financial support.

\section{References}

[1] Fujimoto, Y., Liu, C., Tanaka, Y. and Shintaku, E. (2013) Measurement of Double-Strike Phenomenon of Impulsive Force Using Fluctuating Load Detection Plate. The Japanese Society for Experimental Mechanics, 13, 112-120. (In Japanese)

[2] Liu, C., Tanaka, Y. and Fujimoto, Y. (2015) Viscosity Transient Phenomenon during Drop Impact Testing and Its Simple Dynamics Model. World Journal of Mechanics, 5, 33-41.

[3] Fujimoto, Y., Liu, C., Tanaka, Y., Shintaku, E. and Nakanishi, T. (2015) Thorn-Shape Waveform and Double-Strike Phenomenon Seen in the Impact Force of Soft Material. World Journal of Mechanics, 5, 59-69.

[4] Song, B., Ge, Y., Chen, W.W. and Weerasooriya, T. (2007) Radial Inertia Effects in Kolsky Bar Testing of Extra-Soft Specimens. Experimental Mechanics, 67, 659-670. http://dx.doi.org/10.1007/s11340-006-9017-5

[5] Kwon, J. and Subhash, G. (2010) Compressive Strain Rate Sensitivity of Ballistic Gelatin. Journal of Biomechanics, 43, 420-425. http://dx.doi.org/10.1016/j.jbiomech.2009.10.008

[6] Chen, W., Lu, F. and Winfree, N. (2002) High-Strain-Rate Compressive Behavior of a Rigid Polyurethane Foam with Various Densities. Experimental Mechanics, 42, 65-73. http://dx.doi.org/10.1007/BF02411053

[7] Hoffman, R.L. (1972) Discontinuous and Dilatant Viscosity Behavior in Concentrated Suspensions. I. Observation of a Flow Instability. Transactions of the Society of Rheology, 16, 155-173. http://dx.doi.org/10.1122/1.549250

[8] Bender, J. and Wagner, N.J. (1996) Reversible Shear Thickening in Monodisperse and Bi-disperse Colloidal Dispersions. Journal of Rheology, 40, 889-915. http://dx.doi.org/10.1122/1.550767 


\section{Appendix: Impact Force Waveform of a Spherical Collision with Oil Clay}

Figure A1(a) shows a urethane rubber hemisphere (rubber hardness: 90, diameter: $40 \mathrm{~mm}$ ) fixed to the bottom of the drop hammer and a flat oil clay plate $(T=50 \mathrm{~mm})$. Figure A1(b) shows a drop hammer with a flat bottom and an oil clay prepared into a semi-spherical shape. In both cases, the drop height is $h=380$ mm. Figure A2 shows the measured impact force waveforms. The thorn shape does not appear in the figure. Thus, when the colliding body or the receiving body is not planar, a thorn is less likely to appear in the waveform because the impact area spreads gradually. The start of the impact force of the lower sensor is delayed compared to the upper sensor. This is due to the influence of the mass inertia of the oil clay.

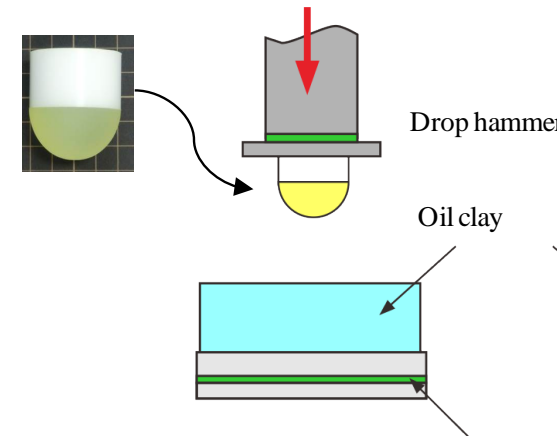

(a)

Lower sensor
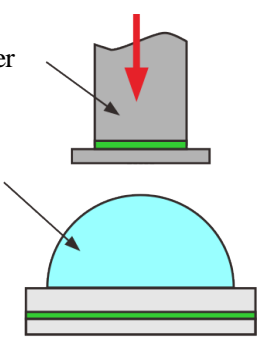

(b)

Figure A1. Experiment to examine spherical collisions. (a) Drop hammer is hemispheical. (b) Clay is hemispheical.

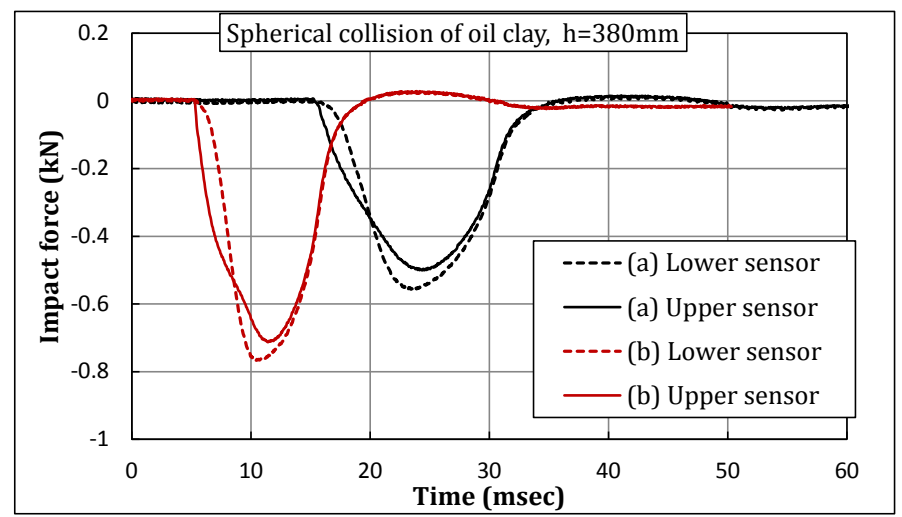

Figure A2. Impact force induced by hammer drop onto an oil clay (spherical collision). 